gear, so that the car runs very quietly. The ad.
vantages of this peculiar type of motor are in the varge and well ventilated armature, giving a powerful torque, and permitting the use of very heavy currents without excessive heating, and, second, in the
simple form of the field wagnets enabling magnetisw to be obtained with comparatively small expenditure of energy. The actual maximum efficiency of the motor chine was designed, however, to give its maximum eff
ciency at something like its a verage output. Fig. 2 shows its efficiency curve at various loads. The motor
being nominally of $20 \mathrm{~h}$. p., its point of maximum efticiency is at about $15 \mathrm{~h}$. p., and even at $15 \mathrm{~h}$. p., its normal load, the efficiency will be seen to be 80 per good all-around commercial efficiency even under wide ly varying loads. The use of a single wotor once geared is in itself a decided advantage, as it both increases the commercial efficiency by avoiding multiplicity of equipment. The motor and the regulating devices together weigh less than 1,590 pounds, with the result ing appliances in this Waddell-Entz car is less than that of the motor equipment of the standard cars used on
the trolley system. This means a very decided saving in power, for one of the besetting sins of the storage battery system has been the excessive weight of equipburned at the power station. Of course the same or desired, although such has not been the usual prac-
tice. The total weight of the copper in the motor is
130 pounds, a very satisfactory result for a motor intended for such exceedingly heavy currents. The regulating appliances of the car are interesting. The plat-
form switch has six steps. Of these the first throws form switch has six steps. Of these the first throws
on the batteries, four in multiple to start the car, the second changes this arrangement to two in multiple, the third throws all the cells in series, while the
fourth and fifth cut out sections of the field, and the

rixth reverses the motor on its lowest speed.
The motors when the car is running down hill can

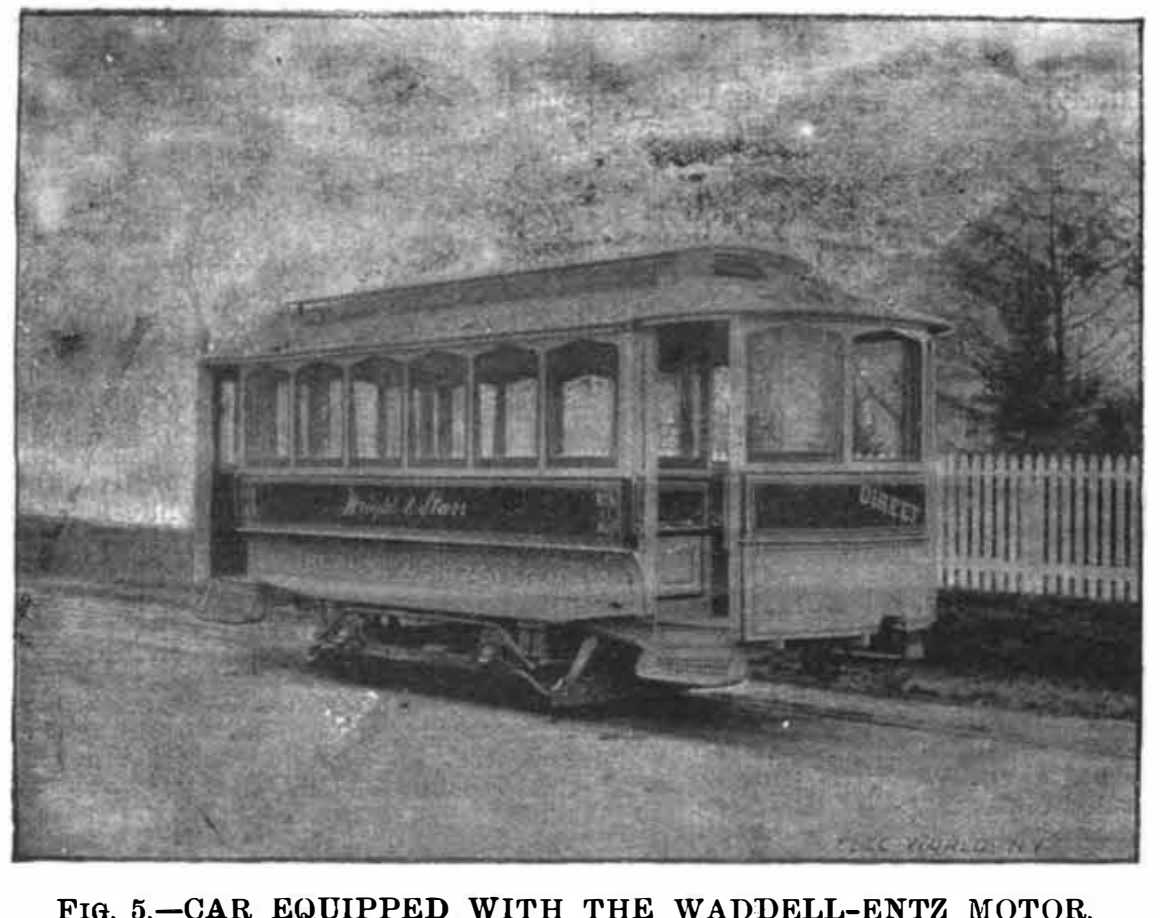

FIG. 5.-CAR EQUIPPED WITH THE WADDELL-ENTZ MOTOR.

operate as dynamos and restore energy to the batteries. his feature of the system is of practical use of course only on roads with perceptible grades, but under such possibly saving as much as 10 per cent. of energy. 'The
road over which the trial trip was made is almost exroad over which the trial trip was made is almost exactly three miles in length, giving a six mile round trip
for the car, and the power required to charge the batteries for the work is very nearly six horse power hours, practically one horse power hour per car mile. greater than this, sufficient for perhaps half a day of ordinary service. This result of one horse power hour per car mile at the power station is immensely satisusually obtained by storage battery cars, and compares on trolley roads, which, however, it should be said, have heavier grades and a heavier car equipment than the one we are considering. The trial trip was a
thorough and complete success. The car ran smoothly thorough and complete success. The car ran swoothly and regularly, and its motion down grades was reported tootors and the braking action of their use as dynamos. just mentioned. Fig. 5 shows a view of the complete 4 a diagram of the output required at each point of 4 a diagram of the output required at each point of
the test run over the road. The readings were taken the test run over the road. The readings were taken
with Weston ammeters and voltmeters each ten seconds and the resilt shows clearly the ability of the batteries to stand a very heavy output without serious drop in represent, of course, periods during which energy was
being restored to the battery. It will be seen that the being restored to the battery. It will be seen that the
running speed in this test was 10 miles per hour, the running speed in this test was 10 miles per hour, the this alkaline storage battery motor system seems to be a very useful addition to the methods of electric tracfor some time in the way of proper equipment for city service. Of course time alone will tell its success when but the results obtained are certainly such as to warvocates of storage battery traction. - Ellectrical World.
THE ANALYSIS OF DEGRAS.

$$
\text { By F. Simand. }
$$

GENUINE degras contains a substance described by
ean as resembling a resin, which the present autho of which the producer or substance of the degras depend the quantity the proportion of it in any sample, the higher is the tate is of a brownish black color, but after purificacen, dissolves very readily in alkalies and ammonia and is precipitated from these solutions by acids in the specialiy when it contains a little acid, dissolves the hol, glacial acetis acid, and aniline, almost ine in alcoher, and quite insoluble in light petroleum and benne. It does not melt on heating, and is chiefly found freparing which it appears to be formed. It occurs however, in greater or smaller amounts in almost al
crain oils, and is of great value for tanning purposes. In order to determine the amount of this degras he amount of water contained in it, are saponified on the water bath with 5-6gus. of caustic soda dissolved
in about 10 c.c. of water and $50-60$ c.c. of alcohol, loss of alcohol being hindered as much as possible by a smal funnel placed in the neck of the flask. As soon
as the whole is dissolved, the alcohol is d riven off and the saap dissolved in water, the fatty acids, together ydrochloric acid. The liquid is then heated until the atty acids form a clear layer at the top, the whole allowed to cool, and the aqueous solution poured away contains a little of the degras substance in solution, and ated. The fatty acids, which contain the degras subtance, are extracted several times with boiling wate to remove hydrochloric acid and the wash water neutralized with ammonia and added to the first filtrate.
These combined washings are evaporated to dryness,

the residue dissolved in a little water, and a slight substance is filtered off, washed until free from hydroacids, etc., which have in the meantime been dried at $105^{\circ}$ (this drying is not absolutely necessary). The united inass is placed in an Erlenweyer flask and cov$70^{\circ}$. The fatty acids dissolve, whereas the degras substance and a suall amount of albumenoid matter remain undissolved. The liquid is filtered, the residue
washed with light petroleum, the filtrate being evaporated and the fatty acids weighed. The residual mass,
which was insoluble in the petroleum, is treated with which was insoluble in the petroleum, is treated with
hot alcohol, in which the albumenoid matter is insoluhot alcohol, in which the albumenoid matter is insolu-
ble, the liquid filtered and the degras substance finally tion of the alcohol.

A sample of degras can only be considered genuine degras substance, the amount of water present being 20 per cent. In some cases as much as 16-17 per cent. To deter

are weighed into a tared of water, 25 grms. of degras with a short thermometer to be used as a stirring rod w0-100 grus. of train oil added to render the mass more fluid, and to prevent spurting, and the whole heated on a wire gauzeto $105^{\circ} \mathrm{C}$. until no more gas bubbles are
given off; the loss of weight is dueto water. A certain amount of other volatile matter is lost with the water, The train oil or other oil (vaseline oil is not well adapted for the purpose) which is used must have been previously heated to $105^{\circ}$, if necessary, with the addition of water until all volatile matter has been removed. The amount of water varies from 15-20 per cent. in
produets obtained by the French process, and from $20-$ 0 per cent in leather dre rench procesa,

per cent. in leather dressers' degras.
The ash is determined by heating 25 grms. of degras until all the water has been driven off, the mass being
continually stirred with a glass rod, cleaning the rod from adhering fat by a piece of filter paper, lighting
the degras by means of this paper, and heating the residue to redness.

It varies from a few hundredths per cent. in the
French product to about three per cent. in leather French product
If, through careless work, any sodium or ammonium presence being shown by the formation of crystals he whole is dissolved in a little ammonia, water adder, and then a slight excess of hydrochloric or sulphuric.
acid. The precipitate is washed on a weighed filter paper with cold water and weighed.

The author has investigated the properties of this as er, is readily soluble in petroleum and with difficulty in alcohol; on saponification and decomposition with acid, a substance resembling the fatty acids, and, like The emulsifying power of degras depends upon The amount of degras substance contained in it clude that the compound of this degras substance which occurs in degras is either decomposed hy liberaced degras substance, or is taken up unmore intimately and firmly combined with the deterer is saturated with tannin, and therefore shows tendency to combine with another substance. The action of the degras substance in genuine degras, thereare tanned soft and contain fiber, which are to a certain extent crude and in a position to combine with it;
the prepared leather will then be very sof $t$ and resemble chamois leather

In connection with his examination of genuine deras, the author also communicates the method adopted he course of such an examination he determines (1) The degras substance, arising from genuine degras,
present in the sample or from train oil : (2) Suint; (3) present in the sample or from train oil ; $(2)$ Suint ; (3)
Vaseline oil ; (4) Resin, colophonium. Water and ash are determined as in the genuine products.
and

ESTIMATION OF THE DEGRAS SUBSTANCE.

5-10 grms. or more of the sample are saponified in a flask, the fatty acids precipitated by hydrochloric alcohol and estimated as already described.

\section{ESTIMATION OF WOOL FAT.}

The ethereal solution is placed in a weighed flask, the parts of acetic anhydride for 1-2 hours, the flask being connected with an inverted condenser. Water is then added and the precipitate repeatedly extracted with water to remove acetic acid, af ter which it is dried, and
the mixture of acetic ethers of the fatty acids and of cholesterin, etc., thus obtained dissolved in 15 parts of boiling alcohol (75-150 c.c.), cooled, filtered from the again dissolved in 15 parts of boiling alcohol, to remove vaseline oil as completely as possible. The cholesteryl acetate is then dissolved in ether, the ether evaporated
from the clear solution, and the residue weighed and from the clear solution, and the residue weighed and The author has examined 7 samples of commercial wool fat, in order to ascertain how much cholesteryl
acetate these yield. He found a minimum of 7.59 , and a maximum of $19 \cdot 43$, the average being 14.05 per cent. According to this the factor for calculating the amount
of wool fat would be $10 \cdot 42,5 \cdot 15$, or $7 \cdot 11$. Thus, for exof wool fat would be $10 \cdot 42,5 \cdot 15$, or $7 \cdot 11$. Thus, for ex-
ample, $0 \cdot 4 \mathrm{grm}$. cholesteryl acetate obtained from 10 4rms. of cent. of wool fat, or taking the average nunber, 2844 per cent. Chemists intrusted with such analysis will be able to tell from the appearance of the melting point, whether 20 or 40 per cent. are present. In any case the presence of wool fat can be detecterl
with certainty and estimated with sufficient accurac. f further indication of wool fat in a mixture of fats is ot solidify, the lustrous and non-crystalline if this does the solid fatty acids obtained on saponification: the smell of wool fat, especially when the fat is rubbed on the hands, can also be easily recognized. It must be
borne in mind that small quantities of cholesterin also ccur in genuine degras and in fish oils; the fatty or, from some of the fish oils, not at all, so that any The vaseline oil is estimated by evaporating off the alcoholic solution, which contains the acetylated fatty acids, the vaseline oil. and the resin. The residue is saponified with caustic soda, glycerine added (Nitrete, and the vaseline oil extracted by shaking with light pesoap, which is removed by agitating it with water,
after the removal of the petroleum, adding a little
magnesium sulphate, which forms an insoluble soap, magnesium sulphate, which forms an insoluble soap, and again extracting with light petroleum.

ESTIMATION OF COLUPHONIUM.

The residual soap solution is decomposed with cids and resin dissolved in alcohoi, the alcohol evapo rated off in a weighed vessel, and the residue weighed. The resin acid is then determined in an aliquot part
by the method of $\mathrm{V}$. Hubel and Stadler. The silver salts are extracted with ether, in which the salt of the resin acid is soluble, and the latter is then decomposed the resin can then be determined by evaporating The ether. The author examined one sample from a well-
known German works, which from its price should have been genuine chamois degras, but really consisted
of water 15.8 per cent.. wool fat not less than 20 per cent., vasel ine oil 971 per cent., resin 10 per cent., fish oil 44.46 per cent., in which about 1 per cent. of degras substiance is contained. The article was, therefore, an
artificial product, and had never been near chamois leather. wool fat 35 per cent. (about), vaseline oil 10 per cent.,
genuine anhydrous degras abount 25 per ccnt.. fish oil,
palm oil, etc., 12.31 per cent. - Chemiker Zeitung. 